\title{
Preliminary Results in Decentralized Estimation for Single-Beacon Acoustic Underwater Navigation
}

\author{
Sarah E. Webster \\ Dept. of Mechanical Engineering \\ Johns Hopkins University \\ Baltimore, MD 21218 \\ Email: swebster@jhu.edu
}

\author{
Louis L. Whitcomb \\ Dept. of Mechanical Engineering \\ Johns Hopkins University \\ Baltimore, MD 21218 \\ Email: 1lw@jhu.edu
}

\author{
Ryan M. Eustice \\ Dept. of Naval Arch. and Marine Eng. \\ University of Michigan \\ Ann Arbor, MI, 48109 \\ Email: eustice@umich.edu
}

\begin{abstract}
We report a decentralized, vehicle-based extended information filter designed to enable single-beacon acoustic navigation of multiple underwater vehicles. In single-beacon navigation, ranges from a single reference beacon to a moving underwater vehicle are used in addition to the vehicle's inertial navigation sensors to perform absolute (as opposed to relative) localization and navigation. For this implementation we assume a moving reference beacon from which range measurements are calculated using asynchronous acoustic data broadcasts that also contain information about the reference beacon's position and recent sensor measurements. We assume that other than these acoustic data transmissions the vehicle has no knowledge of the beacon's position or sensor measurements. This implementation allows the simultaneous navigation of multiple vehicles within acoustic range of the reference beacon. Within this framework we show that the decentralized information filter yields identical results to a centralized extended Kalman filter at the instant of each range measurement; in addition we show that between range measurements the results from the two filters differ only by linearization errors. We compare the state estimation results of the decentralized information filter to that of a centralized extended Kalman filter using a simulated data set.
\end{abstract}

\section{INTRODUCTION}

This paper reports the derivation of a decentralized extended information filter (DEIF), for single-beacon one-way-traveltime (OWTT) navigation [6], [7], [26]. This extended information filter is designed to run locally on a submerged vehicle with real-time access to the vehicle's navigation sensors and infrequent, asynchronous access to acoustic broadcasts from a moving reference beacon. The DEIF does not have access to real-time global positioning system (GPS) measurements from the reference beacon or any other information except information that is received acoustically.

Our goal is to enable high-precision absolute navigation of multiple underwater vehicles over length scales of $\mathcal{O}(1-$ $100 \mathrm{~km}$ ). Conventional "dead-reckoning" navigation solutions rely on strap-down sensors such as Doppler velocity logs (DVLs) and inertial measurement units (IMUs) to measure the vehicle attitude, linear and angular velocity, and acceleration. These measurements can be integrated to estimate relative change in vehicle position but yield an estimate of local displacement with errors that are unbounded over time. In order to achieve bounded-error navigation, additional navigation information is required from an absolute georeferenced source.
Traditional methods such as ultra short baseline navigation (USBL) and some implementations of long baseline navigation (LBL) suffer from a lack of scalability where the rate at which multiple vehicles can receive navigation updates decreases linearly as the number of vehicles in the water increases [13]. In addition, LBL navigation requires external, fixed reference beacons that limit the vehicle's navigable range to $5-10 \mathrm{~km}$ from the beacon. In contrast, the data broadcasts used in OWTT navigation enable the navigation of any vehicle within acoustic range and the use of a single, moving reference beacon eliminates the need for multiple, fixed beacons and their associated cost and range limitations.

Within the context of single-beacon navigation, the decentralized approach provides a flexible, scalable solution for vehicle navigation. Navigation algorithms that rely on a centralized observer suffer from the severely limited bandwidth and high latency associated with underwater acoustic communication in comparison to typical land-based radio frequency communication networks [14]. Given the speed of sound in water $(\sim 1500 \mathrm{~m} / \mathrm{s})$, transmitting acoustic data over length scales on the order of kilometers results in latency on the order of seconds. And though the bandwidth of acoustic modem technology has increased dramatically in recent years, achieving throughput of up to $2400 \mathrm{bps}$ [22], operationally the average throughput is on the order of 10-50 bps due to the low duty cycle with which these messages are typically transmitted during at-sea operation. The proposed information filter lends itself to single-beacon navigation because the sparse structure and additive nature of measurements updates in the EIF, described in Section III, requires a minimal quantity of information to be transmitted from the beacon to the vehicle.

This paper is organized as follows: Section II describes previous work in the area of single-beacon navigation and the use of information filters in decentralized estimation. Section III presents the derivation of the decentralized extended information filter and shows that it produces identical results to that of a centralized extended Kalman filter immediately following each range measurement. Section IV presents the results of the simulation used to validate the DEIF. Section V concludes. 


\section{Previous Work}

Single-beacon navigation relies on range measurements from a single, georeferenced beacon to provide an absolute position reference. Previous work in the area of single-beacon navigation is extensively reviewed in [26]. This section reviews some of the references most relevant to this paper. Navigation with a single, fixed beacon whose position is known a priori has been reported using several different estimation techniques - a least squares approach in [21] and [1], and a vehicle-based EKF in [16] and [11]. Navigation with respect to a moving beacon whose position is not known a priori is reported in [17] using a nonlinear least mean square method; in [7] using a maximum likelihood method; and in [26] using a centralized EKF. However, each of these methods, as reported, is only structurally tractable for post-processing, though the authors of [17] suggest improvements that would allow for real-time implementations.

Decentralized estimation in the context of underwater communication and navigation faces unique constraints in terms of low bandwidth and high latency, which renders many of the decentralized estimation solutions from land-based applications unsuitable. Until recently little research has been done on the topic of decentralized estimators and multi-vehicle navigation in the field of underwater robotics. However, as the cost of vehicles has decreased and their reliability improved, the increased interest in multi-vehicle operations within the ocean science community has precipitated new research in this area. In [2] the author presents a method that allows a vehicle running a Bayes estimator to use range and position information broadcast from one or more moving beacons. This work expands on the moving long baseline concept in [24] to encompass multiple range sources and real-time operation. The authors of [18] address a similar concept to moving long baseline and compare the use of the Kalman filter to the particle filter on the vehicle's localization performance.

The estimation algorithm that most closely resembles our work is [8], where the authors rely on a single moving georeferenced beacon to support the localization of multiple vehicles through asynchronous acoustic broadcasts. The main difference between the algorithm presented in [8] and the algorithm presented herein, is that [8] employs a vehiclebased EKF and performs range measurement updates using the absolute position and covariance broadcast from the reference beacon. The benefit of this formulation is that the algorithm is trivially robust to packet loss, however excluding the reference beacon position from the state vector of the filter makes the results difficult or impossible to compare analytically to a centralized filter. In contrast, the decentralized algorithm reported herein recreates the results of a centralized EKF that has access to measurements from both the vehicle and the beacon's navigation sensors. In addition, by keeping both vehicle and ship states in the filter, range measurement updates do not risk over confidence in and correlation of observations. In [2], the work upon which [8] is based, a multi-hypothesis strategy is employed to avoid over-confidence by preventing measurement data from being incorporated multiple times.

Finally, we look specifically at the use of the information filter for navigation in the field of robotics. Derived in detail in [19], the extended information filter has been employed for vehicle navigation [3] and SLAM algorithms [20], [23] in the context of land-based robotics. In the context of underwater vehicles, the EIF is most widely used for coordinated control, but there are a few examples of the EIF being employed in SLAM algorithms [4], [5]. To the best knowledge of the authors, the present paper is the first to formulate and test an extended information filter in the context of decentralized single-beacon navigation for underwater vehicles.

\section{Derivation of The Decentralized EXTENDED INFORMATION FILTER}

In this section we present a brief review of the update and measurement equations for the extended information filter followed by a derivation of the vehicle-based decentralized extended information filter (DEIF). The implementation of the DEIF relies on two separate filters, both of which process sensor data causally and asynchronously. The information filter on the ship has access to ship sensor data but not range measurements (we will use the term ship to refer to the reference beacon for the remainder of this derivation). The ship-based filter is used to calculate the change in the ship's information vector and information matrix between acoustic broadcasts, and this delta information is acoustically transmitted to the vehicle. As described in [25], the ship-tovehicle range is measured using the one-way-travel-time of the acoustic broadcast. The DEIF on the vehicle has real-time access to vehicle sensor data and the asynchronous acoustic broadcasts from the ship, but does not have access to the ship's sensor measurements apart from the acoustic broadcasts. A centralized EKF (CEKF), which has simultaneous, realtime access to both vehicle and ship sensor data, provides the benchmark for the performance of the DEIF. Figure 1 shows a schematic of the delta ship information transmitted from the ship to the vehicle.

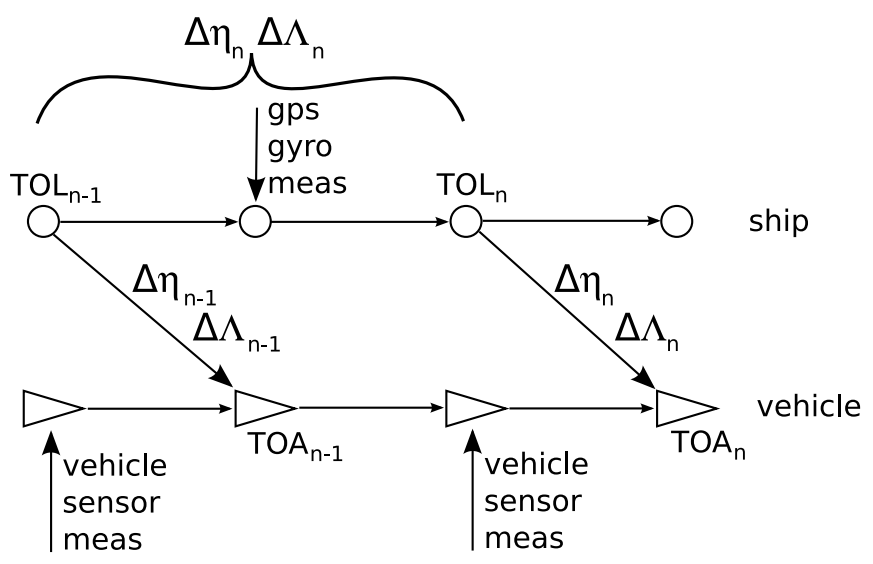

Fig. 1. A schematic of the information contained in the range packet acoustically transmitted from the ship to the vehicle. 


\section{A. Extended Information Filter Equations}

The EIF is characterized by the information matrix, $\boldsymbol{\Lambda}$, and the information vector, $\boldsymbol{\eta}$, which can be defined in terms of the mean, $\boldsymbol{\mu}$, and covariance, $\boldsymbol{\Sigma}$, of the state vector, $\boldsymbol{x}$, [12], [19], as

$$
\begin{aligned}
\boldsymbol{\Lambda} & =\boldsymbol{\Sigma}^{-1} \\
\boldsymbol{\eta} & =\boldsymbol{\Lambda} \boldsymbol{\mu}
\end{aligned}
$$

where

$$
\begin{aligned}
\boldsymbol{\mu} & =E[\boldsymbol{x}] \\
\boldsymbol{\Sigma} & =E\left[(\boldsymbol{x}-\boldsymbol{\mu})(\boldsymbol{x}-\boldsymbol{\mu})^{\top}\right] .
\end{aligned}
$$

1) Process Prediction: For the general process prediction equations we consider a state vector with two states in it, the current state $\boldsymbol{x}_{k}$ and a previous state $\boldsymbol{x}_{p}$, so that

$$
\boldsymbol{x}_{k \mid k}=\left[\begin{array}{c}
\boldsymbol{x}_{k} \\
\boldsymbol{x}_{p}
\end{array}\right]
$$

and

$$
\begin{aligned}
\boldsymbol{\Lambda}_{k \mid k} & =\left[\begin{array}{ll}
\boldsymbol{\Lambda}_{k k} & \boldsymbol{\Lambda}_{k p} \\
\boldsymbol{\Lambda}_{p k} & \boldsymbol{\Lambda}_{p p}
\end{array}\right] \\
\boldsymbol{\eta}_{k \mid k} & =\left[\begin{array}{l}
\boldsymbol{\eta}_{k} \\
\boldsymbol{\eta}_{p}
\end{array}\right] .
\end{aligned}
$$

The process prediction equations for the information filter, which represent the distribution $p\left(\boldsymbol{x}_{k+1}, \boldsymbol{x}_{p} \mid \mathbf{Z}^{1: k}, \mathbf{U}^{1: k+1}\right)$, are

$$
\begin{aligned}
\boldsymbol{\Lambda}_{k+1 \mid k} & =\left[\begin{array}{cc}
\Psi_{k} & \boldsymbol{Q}_{k}^{-1} \boldsymbol{F}_{k} \boldsymbol{\Omega}_{k}^{-1} \boldsymbol{\Lambda}_{k p} \\
\boldsymbol{\Lambda}_{k p} \boldsymbol{\Omega}_{k}^{-1} \boldsymbol{F}_{k}^{\top} \boldsymbol{Q}_{k}^{-1} & \boldsymbol{\Lambda}_{p p}-\boldsymbol{\Lambda}_{p k} \boldsymbol{\Omega}_{k}^{-1} \boldsymbol{\Lambda}_{k p}
\end{array}\right] \\
\boldsymbol{\eta}_{k+1 \mid k} & =\left[\begin{array}{c}
\boldsymbol{Q}_{k}^{-1} \boldsymbol{F}_{k} \boldsymbol{\Omega}_{k}^{-1} \boldsymbol{\eta}_{k}+\Psi_{k}\left(\boldsymbol{f}\left(\boldsymbol{\mu}_{k \mid k}, \boldsymbol{u}_{k+1}\right)-\boldsymbol{F}_{k} \boldsymbol{\mu}_{k \mid k}\right) \\
\boldsymbol{\eta}_{p}-\boldsymbol{\Lambda}_{p k} \boldsymbol{\Omega}_{k}^{-1} \boldsymbol{\eta}_{k}^{*}
\end{array}\right]
\end{aligned}
$$

where $\mathbf{Z}^{1: k}$ are the vehicle sensor measurements up to time $k$ and $\mathbf{U}^{1: k+1}$ are the control inputs up to time $k+1, \boldsymbol{f}(\cdot)$ is the nonlinear process model, $\boldsymbol{F}_{k}$ is the Jacobian, $\boldsymbol{Q}_{k}$ is the covariance of the discretized process model noise, and

$$
\begin{aligned}
\Psi_{k} & =\left(\boldsymbol{Q}_{k}+\boldsymbol{F}_{k} \boldsymbol{\Lambda}_{k k}^{-1} \boldsymbol{F}_{k}^{\top}\right)^{-1} \\
\boldsymbol{\Omega}_{k} & =\boldsymbol{\Lambda}_{k k}+\boldsymbol{F}_{k}^{\top} \boldsymbol{Q}_{k}^{-1} \boldsymbol{F}_{k} \\
\boldsymbol{\eta}_{k}^{*} & =\boldsymbol{\eta}_{k}-\boldsymbol{F}_{k}^{\top} \boldsymbol{Q}_{k}^{-1}\left(\boldsymbol{f}\left(\boldsymbol{\mu}_{k \mid k}, \boldsymbol{u}_{k+1}\right)-\boldsymbol{F}_{k} \boldsymbol{\mu}_{k \mid k}\right)
\end{aligned}
$$

as derived in [5], [23].

2) Process Prediction with Augmentation: In equations (8) and (9), the current state at time $k$ is propagated to time $k+1$, so that

$$
\boldsymbol{x}_{k+1 \mid k}=\left[\begin{array}{c}
\boldsymbol{x}_{k+1} \\
\boldsymbol{x}_{p}
\end{array}\right] .
$$

If instead we augment the state vector to include the state at time $k+1$ in addition to the original states,

$$
\boldsymbol{x}_{k+1 \mid k}=\left[\begin{array}{c}
\boldsymbol{x}_{k+1} \\
\boldsymbol{x}_{k} \\
\boldsymbol{x}_{p}
\end{array}\right],
$$

the process prediction equations, which represent the distribution $p\left(\boldsymbol{x}_{k+1}, \boldsymbol{x}_{k}, \boldsymbol{x}_{p} \mid \mathbf{Z}^{1: k}, \mathbf{U}^{1: k+1}\right)$, have a very different structure, [5],

$$
\begin{aligned}
\boldsymbol{\Lambda}_{k+1 \mid k}= & {\left[\begin{array}{ccc}
\boldsymbol{Q}_{k}^{-1} & -\boldsymbol{Q}_{k}{ }^{-1} \boldsymbol{F}_{k} & \mathbf{0} \\
-\boldsymbol{F}_{k}^{\top} \boldsymbol{Q}_{k}^{-1} & \boldsymbol{F}_{k}^{\top} \boldsymbol{Q}_{k}^{-1} \boldsymbol{F}_{k}+\boldsymbol{\Lambda}_{k k} & \boldsymbol{\Lambda}_{k p} \\
\mathbf{0} & \boldsymbol{\Lambda}_{p k} & \boldsymbol{\Lambda}_{p p}
\end{array}\right] } \\
\boldsymbol{\eta}_{k+1 \mid k}= & {\left[\begin{array}{c}
\boldsymbol{Q}_{k}^{-1}\left(\boldsymbol{f}\left(\boldsymbol{\mu}_{k \mid k}, \boldsymbol{u}_{k+1}\right)-\boldsymbol{F}_{k} \boldsymbol{\mu}_{k \mid k}\right) \\
\boldsymbol{\eta}_{k}-\boldsymbol{F}_{k}^{\top} \boldsymbol{Q}_{k}^{-1}\left(\boldsymbol{f}\left(\boldsymbol{\mu}_{k \mid k}, \boldsymbol{u}_{k+1}\right)-\boldsymbol{F}_{k} \boldsymbol{\mu}_{k \mid k}\right) \\
\boldsymbol{\eta}_{p}
\end{array}\right] }
\end{aligned}
$$

using the same definitions as in (8) and (9). Equation (15), can be written as the sum of two matrices-one containing the process prediction information and the other containing the previous information matrix.

$$
\begin{array}{r}
\boldsymbol{\Lambda}_{k+1 \mid k}=\left[\begin{array}{ccc}
\boldsymbol{Q}_{k}^{-1} & -\boldsymbol{Q}_{k}^{-1} \boldsymbol{F}_{k} & \mathbf{0} \\
-\boldsymbol{F}_{k}^{\top} \boldsymbol{Q}_{k}^{-1} & \boldsymbol{F}_{k}^{\top} \boldsymbol{Q}_{k}^{-1} \boldsymbol{F}_{k} & \mathbf{0} \\
\mathbf{0} & \mathbf{0} & \mathbf{0}
\end{array}\right]+ \\
{\left[\begin{array}{ccc}
\mathbf{0} & \mathbf{0} & \mathbf{0} \\
\mathbf{0} & \boldsymbol{\Lambda}_{k k} & \boldsymbol{\Lambda}_{k p} \\
\mathbf{0} & \boldsymbol{\Lambda}_{p k} & \boldsymbol{\Lambda}_{p p}
\end{array}\right]}
\end{array}
$$

As was first noted in [5], this results in $\boldsymbol{\Lambda}$ having a sparse, block-tridiagonal structure. The sparsity of $\boldsymbol{\Lambda}$ is important in the context of acoustic navigation because it bounds the amount of information that must be acoustically transmitted in order to fully reconstruct the ship's state (see Section IIIB).

3) Measurement Update: The measurement update equations for the extended information filter are

$$
\begin{aligned}
\boldsymbol{\Lambda}_{k \mid k}= & \boldsymbol{\Lambda}_{k \mid k-1}+\boldsymbol{H}_{k}^{\top} \boldsymbol{R}_{k}^{-1} \boldsymbol{H}_{k} \\
\boldsymbol{\eta}_{k \mid k}= & \boldsymbol{\eta}_{k \mid k-1}+\cdots \\
& \boldsymbol{H}_{k}^{\top} \boldsymbol{R}_{k}^{-1}\left(\boldsymbol{z}_{k}-\boldsymbol{h}\left(\boldsymbol{\mu}_{k \mid k-1}\right)+\boldsymbol{H}_{k} \boldsymbol{\mu}_{k \mid k-1}\right)
\end{aligned}
$$

where $\boldsymbol{z}_{k}$ is the measurement, $\boldsymbol{R}_{k}$ is the covariance matrix of the measurement noise, $\boldsymbol{h}(\cdot)$ is the measurement model, $\boldsymbol{H}_{k}$ is the Jacobian of the measurement with respect to $\boldsymbol{x}$

$$
\boldsymbol{H}_{k}=\left.\frac{\partial \boldsymbol{h}(\boldsymbol{x})}{\partial \boldsymbol{x}}\right|_{\boldsymbol{x}=\mu_{k \mid k-1}}
$$

and $\boldsymbol{\mu}_{k \mid k-1}$ is the mean of the state vector [12], [19], [23].

\section{B. Ship-Based Information Filter}

The DEIF, running on the vehicle, relies on acoustic data transmissions from the ship to make range measurements. The acoustic transmission contains a range packet with ship state information from an independent ship-based information filter that is used to maintain both current and historic ship state estimates. This filter is independent of the vehicle state, vehicle sensor measurements, and range measurements.

We assume a constant-velocity linear kinematic process model for the ship, which, in the experience of the authors, is a reasonable assumption for ship motion in the context of AUV survey operations. For the ship's process model the state vector 
contains the ship's xy-position, heading, and the respective velocities,

$$
\boldsymbol{x}_{s}=\left[x_{s}, y_{s}, \theta_{s}, \dot{x}_{s}, \dot{y}_{s}, \dot{\theta}_{s}\right]^{\top} .
$$

The ship process model is identical to that reported in [26], to which the reader is referred for further details.

1) State Vector Augmentation: The independent ship filter maintains an estimate of the current ship state as well as copies of historic ship states. Each time a new acoustic range packet is broadcast, i.e. at the time-of-launch (TOL), a copy of the current ship state is appended onto the ship state vector. This results in a state vector, after $n$ range packets have been transmitted, of the form

$$
\boldsymbol{x}_{s_{k \mid k}}=\left[\begin{array}{c}
\boldsymbol{x}_{s_{k}} \\
\boldsymbol{x}_{s_{T L_{n}}} \\
\vdots \\
\boldsymbol{x}_{s_{T L_{2}}} \\
\boldsymbol{x}_{s_{T L_{1}}}
\end{array}\right]
$$

where we have adopted the following convention: $\boldsymbol{x}_{s_{k \mid k}}$ denotes the entire ship state vector at time $k ; \boldsymbol{x}_{s_{k}}$ is the current ship state; and $\boldsymbol{x}_{s_{T O L_{n}}}$ is the ship state when the $\mathrm{n}^{\text {th }}$ range packet was broadcast. Note that we are using ${ }^{\top}$ for the matrix transpose operator. Because in practice it is undesirable, and unnecessary, for the state vector to grow without bound, we marginalize out [5] the oldest historic ship states in order to maintain a fixed-length state vector.

2) Acoustic Data Packets: What differentiates the DEIF from other estimators used in decentralized single-beacon navigation is the information that is transmitted with the range measurements and how that information is incorporated into the decentrialized vehicle navigation filter in conjunction with the range measurement. To initiate a range measurement, the ship broadcasts an acoustic data packet, or range packet, containing information about the ship state. In other formulations of single-beacon navigation, such as [2] and [8], the range packet contains the mean and covariance of the ship's current $\mathrm{x}-\mathrm{y}$ position, which is used by the filter on the vehicle to perform a range measurement update. This approach, while certainly reasonable, does not contain enough information to allow the vehicle to recreate the global state of the system, including full state recovery of both the vehicle and the ship.

In this formulation, the range packet contains the change in $\boldsymbol{\Lambda}_{s}$ and $\boldsymbol{\eta}_{s}$ between the time of the current acoustic broadcast, $\mathrm{TOL}_{n}$, and the time of the previous acoustic broadcast, $\mathrm{TOL}_{n-1}$,

$$
\begin{aligned}
\Delta \boldsymbol{\Lambda}_{T O L_{n}} & =\boldsymbol{\Lambda}_{s_{T O L_{n}}}-\boldsymbol{\Lambda}_{s_{T O L_{n-1}}} \\
\Delta \boldsymbol{\eta}_{T O L_{n}} & =\boldsymbol{\eta}_{s_{T O L_{n}}}-\boldsymbol{\eta}_{s_{T O L_{n-1}}}
\end{aligned}
$$

where, for conformability, $\boldsymbol{\Lambda}_{s_{T O L_{n-1}}}$ and $\boldsymbol{\eta}_{s_{T O L_{n-1}}}$ have been padded with zeros to match the size of $\boldsymbol{\Lambda}_{s_{T O L_{n}}}$ and $\boldsymbol{\eta}_{s_{T O L_{n}}}$ respectively. These range packets are reassembled subsea in the DEIF.

The contribution to $\Delta \boldsymbol{\Lambda}_{T O L_{n}}$ and $\Delta \boldsymbol{\eta}_{T O L_{n}}$ from the ship sensor measurement updates, (19), and prediction with aug- mentation, (17), are independent of other measurements, because of the additive nature of the respective operations and the linear ship process model. As a result, the $\Delta \boldsymbol{\Lambda}_{T O L_{n}}$ and $\Delta \boldsymbol{\eta}_{T O L_{n}}$ calculated from the ship-based filter, which has no knowledge of the vehicle and is not subject to range measurement updates, is identical to what the DEIF would have calculated if it was performing the ship process predictions and ship sensor measurement updates even though the ship states in the DEIF have been modified by prior range measurements. This is discussed in more detail in Section III-C.

\section{Vehicle-Based Decentralized Extended Information Filter}

The DEIF uses a constant velocity nonlinear process model with a 12 degree-of-freedom (DOF) state vector,

$$
\boldsymbol{x}_{v}=\left[\boldsymbol{s}^{\top}, \boldsymbol{\varphi}^{\top}, \boldsymbol{v}^{\top}, \boldsymbol{\omega}^{\top}\right]^{\top}
$$

where $s$ is the local-level vehicle pose in the local frame, $\varphi$ is the local-level vehicle attitude (Euler roll, pitch, heading), $\boldsymbol{v}$ is the body-frame linear velocity, and $\boldsymbol{\omega}$ is the body-frame angular velocity. The vehicle process model is identical to that reported in [26], to which the reader is referred for further details.

1) DEIF State Vector: In addition to the current vehicle state, the DEIF maintains a copy of the historic ship states reconstructed from the delta information acoustically broadcast from the ship. As a result, the DEIF state vector consists of two parts, the current vehicle state and the historic ship states,

$$
\boldsymbol{x}_{v_{k \mid k}}=\left[\begin{array}{c}
x_{v_{k}} \\
x_{s_{T O L_{n}}} \\
\vdots \\
x_{s_{T O L_{1}}}
\end{array}\right]
$$

where we adopt the convention that $\boldsymbol{x}_{v_{k \mid k}}$ denotes the entire vehicle state vector at time $k ; x_{v_{k}}$ is the current vehicle state; and $x_{s_{T O L_{n}}}$ is the ship state when the $\mathrm{n}^{\text {th }}$ range packet was broadcast. Note that by definition, because acoustic broadcasts have a non-negligible time delay associated with them, the DEIF state vector cannot contain information about the current ship state, it can only contain information about historic ship states.

The reconstructed ship states are not subjected to process predictions or measurement updates other than range measurements, because the DEIF has no knowledge of the ship process model or measurement from ship sensors. The ship states remain unchanged over time except for range measurement updates and when new information from acoustic broadcasts is incorporated.

2) Incorporating Delta Ship Information: At the time-ofarrival (TOA) of a range packet at the vehicle, the delta ship information included in each range packet is incorporated in the DEIF, and then the range measurement update is performed. The delta ship information is incorporated into the DEIF simply by addition, in the analogous operation to (23) 
and (24):

$$
\begin{aligned}
\boldsymbol{\Lambda}_{v_{T O A_{n}}} & =\overline{\boldsymbol{\Lambda}}_{v_{T O A_{n}}}+\Delta \boldsymbol{\Lambda}_{T O L_{n}} \\
\boldsymbol{\eta}_{v_{T O A_{n}}} & =\overline{\boldsymbol{\eta}}_{v_{T O A_{n}}}+\Delta \boldsymbol{\eta}_{T O L_{n}}
\end{aligned}
$$

where $\overline{\boldsymbol{\Lambda}}_{v_{T O A_{n}}}$ is the information matrix before the delta ship information is incorporated and $\boldsymbol{\Lambda}_{v_{T O A_{n}}}$ is the information matrix after the delta ship information is incorporated, accounting for conformability. As noted in Section III-B.2, the delta ship information encapsulates all of the information that the filter has gained about the ship state since the last range packet was transmitted. The simplicity of this computation is one of the advantages of the information filter.

3) Range Measurement Updates: At the time-of-arrival (TOA) of the range packet, after the delta ship information is incorporated, the range measurement update (19) is performed. The observation model for the range measurement is

$$
z_{k}=\sqrt{\left(\boldsymbol{x}_{v_{x y z}}-\boldsymbol{x}_{s_{x y z}}\right)^{\top}\left(\boldsymbol{x}_{v_{x y z}}-\boldsymbol{x}_{s_{x y z}}\right)}+v_{k}
$$

where $\boldsymbol{x}_{v_{x y z}}$ is the vehicle pose at the TOA, $\boldsymbol{x}_{s_{x y z}}$ is the ship pose at the TOL, and $v_{k} \sim \mathcal{N}\left(0, R_{k}\right)$.

\section{CEKF Delayed Measurement Update}

Immediately after performing a range update, the current state of the vehicle recovered by the DEIF is identical to the vehicle state recovered from the CEKF. There are several subtleties in this observation that we address here.

At the TOA of the range packet, the range measurement is made between $\boldsymbol{x}_{v_{T O A}}$ and $\boldsymbol{x}_{s_{T O L}}$. Comparing the probability distributions of the two filters immediately after the range measurement update, we find that they are not identical:

$$
\begin{gathered}
\text { DEIF: } p\left(\boldsymbol{x}_{v_{k}}, \boldsymbol{x}_{s_{T O L}} \mid z_{r n g}^{T O A}, \mathbf{Z}_{v}^{1: T O A}, \mathbf{U}_{v}^{1: T O A}, \mathbf{Z}_{s}^{1: T O L}\right) \\
\text { CEKF: } p\left(\boldsymbol{x}_{v_{k}}, \boldsymbol{x}_{s_{T O L}} \mid z_{r n g}^{T O A}, \mathbf{Z}_{v}^{1: T O A}, \mathbf{U}_{v}^{1: T O A}, \mathbf{Z}_{s}^{1: T O A}\right)
\end{gathered}
$$

where $z_{r n g}^{T O A}$ is the most recent range measurement, $\mathbf{Z}_{v}^{1: T O A}$ are the vehicle sensor measurements up to the TOA, $\mathbf{U}_{v}^{1: T O A}$ are the vehicle control inputs up to the TOA, and $\mathbf{Z}_{s}^{1: T O L}$ are the ship sensor measurements up to the TOL. Note that the CEKF has access to ship sensor measurements between the TOL and the TOA of which the DEIF has no knowledge. This is illustrated in Figure 2 where the CEKF has access to the GPS and gyrocompass measurements made at the ship between the TOL and TOA, while the DEIF does not.

The ramifications of this are that the DEIF performs a range measurement between the current vehicle state and the the best estimate of the ship's state at the TOL given ship sensor measurements only up to the TOL. In contrast, the CEKF performs a range measurement between the current vehicle state and the best estimate of the ship's state at the TOL given ship sensor measurement up to the TOA. The CEKF is essentially performing a smoothing operation on the

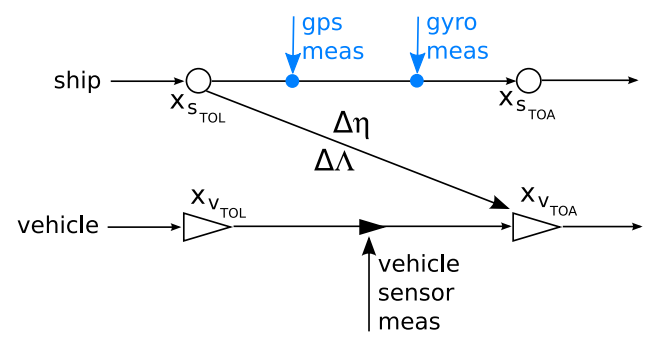

Fig. 2. At the TOA, the CEKF has access to the GPS and gyrocompass measurements made at the ship after the TOL, while the DEIF does not.

ship's state at the TOL, because it has access to additional information from the ship's sensors after the data packet was broadcast at the TOL.

To address this discrepancy we use a two-step delayed measurement update in the CEKF, first performing a measurement update for the range measurement with only ship measurements up until the TOL, (32), and then performing another measurement update for the ship measurements that happened between the TOL and the TOA, (33):

$$
\begin{aligned}
& p\left(\boldsymbol{x}_{v_{k}}, \boldsymbol{x}_{s_{T O L}} \mid z_{r n g}^{\text {TOA }}, \mathbf{Z}_{v}^{1: T O A}, \mathbf{U}_{v}^{1: T O A}, \mathbf{Z}_{s}^{1: T O L}\right) \\
& p\left(\boldsymbol{x}_{v_{k}}, \boldsymbol{x}_{s_{T O L}} \mid z_{r n g}^{\text {TOA }}, \mathbf{Z}_{v}^{1: T O A}, \mathbf{U}_{v}^{1: T O A}, \mathbf{Z}_{s}^{1: T O L}, \mathbf{Z}_{s}^{\text {TOL:TOA }}\right)
\end{aligned}
$$

so that now the DEIF distribution in (30) is identical to the CEKF distribution in (32), without compromising the CEKF's final distribution, i.e. (33) is identical to (31).

Between range measurements, the CEKF and DEIF estimates of the vehicle's state will not be identical because of linearization errors, as seen in (30) versus (31). However, at the instant of each range measurement, through the two-step delayed update, the filters estimates will be identical.

Due to the cumulative nature of this navigation technique, packet loss is an operational concern for real-time implementation. The authors are currently investigating several viable options for addressing the effects of packet loss, including broadcasting redundant range packets or reformulating the delta information in order to mitigate the effect of packet loss.

A final note on this derivation, mentioned briefly in Section III-B.2, is that the ship process model must be linear in order for the delta ship information calculated in the independent ship filter to be identical to a centralized filter. A linear process model guarantees that the process prediction and measurement updates are independent of the ship's current state. This is essential because the independent ship filter will have a different estimate of the ship's current state than a centralized filter, because the centralized filter's estimate is conditioned on previous range measurements. This presents a non-trivial challenge for future work where we would like to incorporate range measurements from other vehicles that will not necessarily have linear process models. 
TABLE I

SIMULATED NAVIGATION SENSORS: SAMPLING FREQUENCY AND NOISE

\begin{tabular}{l|l|l}
\hline \hline Vehicle Sensors & Frequency & Noise \\
\hline & & $h: 0.1^{\circ}$ \\
& & $r p: 0.01^{\circ}$ \\
OCTANS ${ }^{a}$ & $3 \mathrm{~Hz}$ & $0.5^{\circ} / \mathrm{s}$ \\
& & $\dot{r} \dot{p}: 0.25^{\circ} / \mathrm{s}$ \\
\hline depth sensor & $0.9 \mathrm{~Hz}$ & $5 \mathrm{~cm}$ \\
\hline DVL & $3.0 \mathrm{~Hz}$ & $1 \mathrm{~cm} / \mathrm{s}$ \\
\hline modem & every $2.5 \mathrm{~min}$ & $4 \mathrm{~m}$ \\
\hline \hline
\end{tabular}

${ }^{a} h, p$, and $r$ are heading, pitch, and roll respectively; $\dot{h}, \dot{p}$, and $\dot{r}$ are heading, pitch, and roll rates.

\begin{tabular}{l|l|l}
\hline \hline Ship Sensors & Frequency & Noise \\
\hline GPS & $1.0 \mathrm{~Hz}$ & $0.5 \mathrm{~m}$ \\
\hline gyrocompass & $2.0 \mathrm{~Hz}$ & $0.1^{\circ}$ \\
\hline \hline
\end{tabular}

\section{Simulation}

The DEIF is tested using a simulated 6 hours survey at $3800 \mathrm{~m}$ depth. As described in detail in Section III, the DEIF has real-time access to vehicle sensor data and asynchronous acoustic broadcasts from the ship that are used to make range measurements. The DEIF does not have access to the ship's sensor measurements apart from these acoustic broadcasts. To test the validity of the filter, we compare the DEIF estimation results to those obtained with a CEKF, reported in [26], at every time step.

\section{A. Simulation Setup}

For comparison purposes this simulation is designed to mimic the experimental setup of the deep water survey [26]. In the simulated mission presented here, the vehicle drives ten $700 \mathrm{~m}$ tracklines spaced $80 \mathrm{~m}$ apart at a velocity of 0.35 $\mathrm{m} / \mathrm{s}$. The vehicle's depth is constant at $3800 \mathrm{~m}$. The vehicle takes approximately 6 hours to complete the survey, during which time the ship drives around the vehicle's survey area in a diamond pattern at $0.5 \mathrm{~m} / \mathrm{s}$, broadcasting acoustic data packets every 2.5 minutes.

We assume that the ship is equipped with a differential global positioning system (DGPS) receiver and a gyrocompass to measure heading. The vehicle has an OCTANS fiber-optic gyrocompass to measure attitude and attitude rates; a Paroscientific pressure sensor to measure depth; and an RDI Doppler velocity $\log$ (DVL) to measure bottom-referenced velocities. Acoustic modems are used to measure the range between the ship and the vehicle. The vehicle and ship navigation sensors, their sampling frequencies, and the noise statistics for each sensor are given in Table I.

\section{B. Results}

The results of the simulation are shown in Figures 3, 4 and 5. Figure 3 shows the estimated vehicle trajectory overlaid with the 3-sigma covariance of the vehicle position as estimated by the DEIF. The GPS-reported position of the ship as it moves around the vehicle survey area is also shown. Figure 4 shows the difference between the vehicle's true position and the estimate from the DEIF of the vehicle's position over the course of the simulated dive. The error at the end of the dive between the DEIF's estimate of the vehicle position and the true vehicle position is $3.7 \mathrm{~m}$ cross-track and $0.2 \mathrm{~m}$ alongtrack both with $3.1 \mathrm{~m}$ standard deviation. For comparison, had the vehicle relied solely on dead reckoning throughout the dive with no range measurements, the error at the end of the dive between the estimated and true vehicle position would have been $8.8 \mathrm{~m}$ cross-track and $5.6 \mathrm{~m}$ along-track with a 7.8 $\mathrm{m}$ standard deviation. The 3 -sigma error bars are included to show that the filter maintains consistency over the course of the dive.

Comparing the mean of the vehicle's 12 degree-of-freedom (DOF) state vector as estimated by the DEIF versus the CEKF, Figure 5 shows the norm of the difference over the course of the simulation. The lower plot highlights the norm of the difference immediately after a range measurement, as marked by the asterisks. Note that the y-axis on the lower plot has been scaled down by two orders of magnitude to show the precision with which the DEIF is able to reproduce the results of the CEKF. The average difference between the filters across the entire dive is $5.68 \mathrm{e}-3(5.7 \mathrm{~mm})$ in $\mathrm{x}-\mathrm{y}$ position and $3.35 \mathrm{e}-8$ in the other state elements. The average difference immediately after a range measurement is $8.27 \mathrm{e}-5 \mathrm{~m}$ in $\mathrm{x}-\mathrm{y}$ position and $1.70 \mathrm{e}-10$ in the other vehicle states.

\section{Discussion}

As discussed in Section III-D, we expect the DEIF to produce state estimates that are comparable to the CEKF: immediately after each range update the results should be identical; between range updates, the results should differ only due

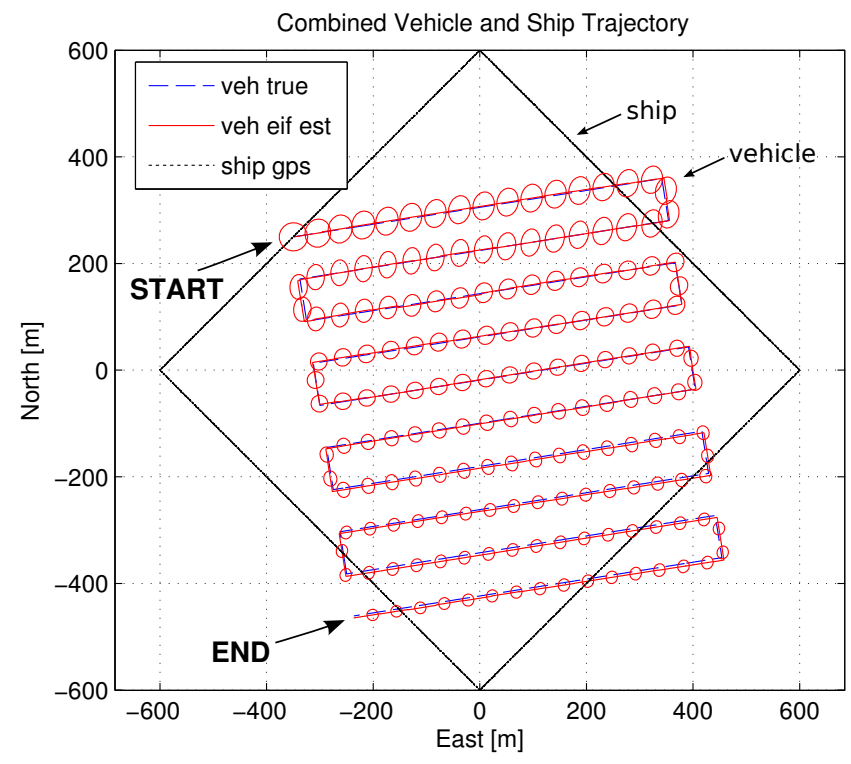

Fig. 3. Ship and vehicle trajectories. The vehicle started in the northwest and proceeded east, ending at the southwest corner of the survey. The ship moved counter-clockwise around the diamond starting at the eastern-most apex. 

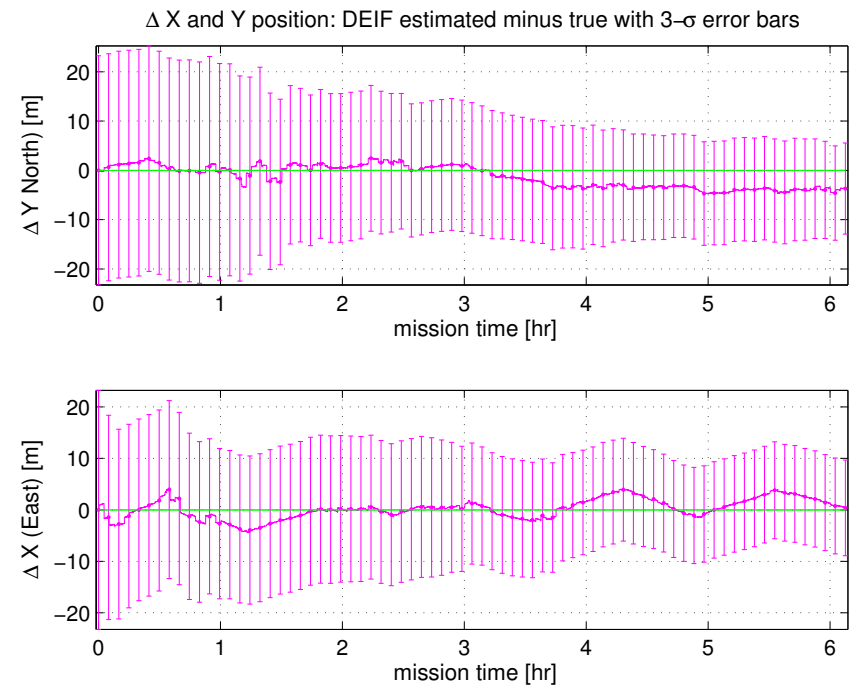

Fig. 4. The difference between the true vehicle position and estimate from the DEIF over time. The error bars are 3 times the standard deviation in each degree of freedom.

to linearization errors. The results from the simulation shown in Figure 5 support this within the tolerance of numerical precision. Over the course of the 6 hour simulated dive the difference in $x-y$ position between the DEIF and the EKF is, on average, $8.27 \mathrm{e}-5 \mathrm{~m}$ immediately after each range update. In addition, the difference between the filters due to linearization errors (averaged over the entire dive) is $5.7 \mathrm{~mm}$ on average with a maximum difference of $4.9 \mathrm{~cm}$.

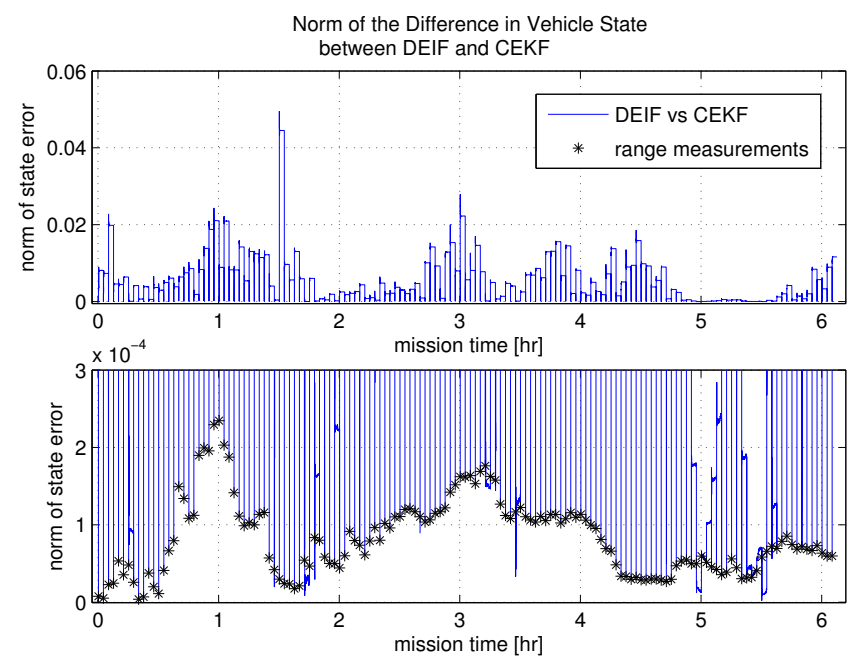

Fig. 5. The sum of the squared error between the mean vehicle position as estimated by the DEIF versus the CEKF.

Because these results are based on a simulated data set, there are several possible discrepancies compared to experimental data. The assumed noise characteristics of the navigation sensors in Table I are used both in the simulation of noisy sensor data and in the measurement models in the DEIF and CEKF. As a result the measurement models exactly predict the performance of the navigation sensors. In addition, the noise model of every sensor is assumed to be Gaussian. While these assumptions may be reasonable for common vehicle navigation sensors that have been tested extensively in the field [15], acoustic range measurements suffer from highlyvariable, non-Gaussian noise sources including multi-path and ray-bending errors. In an attempt to account for this, we use a large assumed variance for the range measurements. In a real-world context outlier filtering of the range data would be necessary.

\section{Conclusions And Future Work}

The structure of the information filter makes it a natural choice for a decentralized implementation. Delayed ship updates are simply additive and require a minimal amount of information to be acoustically transmitted that is well within the function limits of available acoustic modems [9], [10]. In this paper we have derived a vehicle-based extended information filter that is able to estimate a vehicle's state, including $\mathrm{x}-\mathrm{y}$ position, using only vehicle-based inertial navigation sensors and asynchronous acoustic broadcasts from a single, moving, georeferenced beacon. The DEIF is able to locally recreate vehicle state estimates that are commensurate with the results from a centralized extended Kalman filter within a margin of numerical error, and did so over the course of a simulation that is representative of an actual, deep-water survey in both physical scale and the frequency of measurements. In addition, the filter in its current form could be used on multiple underwater vehicles where each vehicle simultaneously receives acoustic data broadcasts from the reference beacon. Given the favorable results in simulation of the DEIF, we look forward to experimentally validating this algorithm and continuing to work towards a full multi-vehicle implementation.

In the future, the natural expansion of this algorithm is to incorporate acoustic broadcasts from other vehicles in addition to broadcasts from the reference beacon. The addition of vehicle-based acoustic broadcasts would generate inter-vehicle range information that could be used to further constrain each receiving vehicle's navigation solution. Incorporating intervehicle ranges presents a number of challenges for continued research, including the nonlinearity of the process models of the vehicles initiating the acoustic broadcast, and the problem of over confidence associated with double counting information passed between the vehicles.

\section{ACKNOWLEDGMENTS}

This work was supported by the National Science Foundation under NSF Awards ATM-0427220, ATM-0428122, and IIS-0746455.

\section{REFERENCES}

[1] P. Baccou and B. Jouvencel, "Homing and navigation using one transponder for AUV, postprocessing comparisons results with long base-line navigation," in Proc. IEEE Int. Conf. Robot. Auto. (ICRA), vol. 4, 2002, pp. 4004-4009. 
[2] A. Bahr, "Cooperative localization for autonomous underwater vehicles," $\mathrm{Ph} . \mathrm{D}$. dissertation, Massachusetts Institute of Technology, Cambridge, MA, USA, February 2009.

[3] M. Bozorg, E. Nebot, and H. Durrant-Whyte, "A decentralised navigation architecture," in Proc. IEEE Int. Conf. Robot. Auto. (ICRA), vol. 4, May 1998, pp. 3413-3418 vol.4.

[4] J. Diosdado and I. Ruiz, "Decentralised simultaneous localisation and mapping for AUVs," in Proceedings of the IEEE OCEANS-Europe Conference and Exhibition, June 2007, pp. 1-6.

[5] R. M. Eustice, H. Singh, and J. J. Leonard, "Exactly sparse delayedstate filters for view-based SLAM," IEEE Trans. Robot., vol. 22, no. 6, pp. 1100-1114, Dec. 2006.

[6] R. M. Eustice, L. L. Whitcomb, H. Singh, and M. Grund, "Recent advances in synchronous-clock one-way-travel-time acoustic navigation," in Proc. IEEE/MTS OCEANS Conf. Exhib., Boston, MA, USA, Sep. 2006, pp. 1-6.

[7] — "Experimental results in synchronous-clock one-way-travel-time acoustic navigation for autonomous underwater vehicles," in Proc. IEEE Int. Conf. Robot. Auto. (ICRA), Rome, Italy, Apr. 2007, pp. 4257-4264.

[8] M. F. Fallon, G. Papadopoulos, and J. J. Leonard, "Cooperative AUV navigation using a single surface craft," in Proc. of Field and Service Robotics, Cambridge, MA, Jul 2009, pp. 1-10.

[9] L. Freitag, M. Grund, J. Partan, K. Ball, S. Singh, and P. Koski, "Multiband acoustic modem for the communications and navigation aid AUV," in Proc. IEEE/MTS OCEANS Conf. Exhib., Washington, D.C., Sep. 2005, pp. 1080-1085.

[10] L. Freitag, M. Grund, S. Singh, J. Partan, P. Koski, and K. Ball, "The WHOI micro-modem: an acoustic communications and navigation system for multiple platforms," in Proc. IEEE/MTS OCEANS Conf. Exhib., Washington, D.C., Sep. 2005, pp. 1086-1092.

[11] A. Gadre and D. Stilwell, "A complete solution to underwater navigation in the presence of unknown currents based on range measurements from a single location," in Proc. IEEE/RSJ Intl. Conf. Intell. Robots Systems (IROS), Edmonton AB, Canada, 2005, pp. 1420-1425.

[12] A. Gelb, Ed., Applied optimal estimation. Cambridge, MA: MIT Press, 1982.

[13] M. Hunt, W. Marquet, D. Moller, K. Peal, W. Smith, and R. Spindel, "An acoustic navigation system," Woods Hole Oceanographic Institution, Tech. Rep. WHOI-74-6, Dec. 1974.

[14] D. Kilfoyle and A. Baggeroer, "The state of the art in underwater acoustic telemetry," Oceanic Engineering, IEEE Journal of, vol. 25, no. 1 , pp. 4-27, Jan 2000.
[15] J. C. Kinsey, R. M. Eustice, and L. L. Whitcomb, "A survey of underwater vehicle navigation: recent advances and new challenges," in IFAC Conf. on Manoeuvring and Control of Marine Craft, Lisbon, Portugal, Sep. 2006.

[16] M. B. Larsen, "Autonomous navigation of underwater vehicles," Ph.D. dissertation, Technical University of Denmark, Denmark, Feb. 2001.

[17] S. McPhail and M. Pebody, "Range-only positioning of a deep-diving autonomous underwater vehicle from a surface ship," Oceanic Engineering, IEEE Journal of, vol. 34, no. 4, pp. 669-677, Oct. 2009.

[18] D. Mirabello, A. C. Sanderson, and D. R. Blidberg, "Comparing Kalman and particle filter approaches to coordinated multi-vehicle navigation," in Proc. Intl. Symp. Unmanned Untethered Subm. Tech. (UUST), Aug. 2007.

[19] A. G. O. Mutambara, Decentralized Estimation and Control for Multisensor Systems. Boca Raton, FL, USA: CRC Press, Inc., 1998.

[20] S. Reece and S. Roberts, "Robust, low-bandwidth, multi-vehicle mapping," in Information Fusion, 2005 8th International Conference on, vol. 2, July 2005, pp. 1319-1326.

[21] A. Scherbatyuk, "The AUV positioning using ranges from one transponder LBL," in Proc. IEEE/MTS OCEANS Conf. Exhib., vol. 3, San Diego, California, 1995, pp. 1620-1623.

[22] S. Singh, S. E. Webster, L. Freitag, L. L. Whitcomb, K. Ball, J. Bailey, and C. Taylor, "Acoustic communication performance of the WHOI micro-modem in sea trials of the Nereus vehicle to 11,000 m depth," in Proc. IEEE/MTS OCEANS Conf. Exhib., Biloxi, MS, Oct 2009, pp. $1-6$.

[23] S. Thrun, Y. Liu, D. Koller, A. Y. Ng, Z. Ghahramani, and H. DurrantWhyte, "Simultaneous localization and mapping with sparse extended information filters," International Journal of Robotics Research, vol. 23, pp. 693-716, 2004.

[24] J. Vaganay, J. Leonard, J. Curcio, and J. Willcox, "Experimental validation of the moving long base-line navigation concept," in Autonomous Underwater Vehicles, 2004 IEEE/OES, June 2004, pp. 59-65.

[25] S. E. Webster, R. M. Eustice, C. Murphy, H. Singh, and L. L. Whitcomb, "Toward a platform-independent acoustic communications and navigation system for underwater vehicles," in Proc. IEEE/MTS OCEANS Conf. Exhib., Biloxi, MS, Oct 2009, pp. 1-7.

[26] S. E. Webster, R. M. Eustice, H. Singh, and L. L. Whitcomb, "Preliminary deep water results in single-beacon one-way-travel-time acoustic navigation for underwater vehicles," in Proc. IEEE/RSJ Intl. Conf. Intell. Robots Systems (IROS), St. Louis, MO, Oct. 2009, pp. 2053-2060. 